\title{
Scale Invariant Theory of Gravitation in Einstein-Rosen Space-Time
}

\author{
Bivudutta Mishra, Pradyumn Kumar Sahoo, Addepalli Ramu \\ Mathematics Group, Birla Institute of Technology and Science, Hyderabad Campus, Andhra Pradesh, Pilani, India \\ E-mail: \{bivudutta, addepallir\}@gmail.com, sahoomaku@rediffmail.com \\ Received June 19, 2010; revised July 19, 2010; accepted July 26, 2010
}

\begin{abstract}
In this paper, we have studied the perfect fluid distribution in the scale invariant theory of gravitation, when the space-time described by Einstein-Rosen metric with a time dependent gauge function. The cosmological equations for this space-time with gauge function are solved and some physical properties of the model are studied.
\end{abstract}

Keywords: Scale Invariant, Space-Time, Perfect Fluid

\section{Introduction}

Several new theories of gravitation have been formulated which are considered to be alternative to Einstein's theory of gravitation. In alternative theories of gravitation, scalar tensor theories proposed by Brans and Dicke [1], Nordvedt [2], Wagoner [3], Ross [4], Dunn [5] and Saez and Ballester [6] are most important among them. In the theory proposed by Brans and Dicke [1] there exists a variable gravitational parameter G. Another theory, which admits a variable $\mathrm{G}$, is the scale covariant theory of Canuto et al. [7]. Dirac [8,9] rebuilt the Weyl's unified theory by introducing the notion of two metrics and an additional gauge function $\beta$. A scale invariant variation principle was proposed from which gravitational and electromagnetic field equations can be derived. It is concluded that an arbitrary gauge function is necessary in all scale-invariant theories.

It is found that the scale invariant theory of gravitation agrees with general relativity up to the accuracy of observations made up now. Dirac [8,9], Hoyle and Narlikar [10] and Canuto et al. [7] have studied several aspects of the scale invariant theory of gravitation. But Wesson's $[11,12]$ formulation is so far the best one to describe all the interactions between matter and gravitation in scale free manner.

Mohanty and Mishra [13] have studied the feasibility of Bianchi type VIII and IX space-times with a time dependent gauge function and a matter field in the form of perfect fluid. In that paper, they have constructed a radiating model of the universe for the feasible Bianchi type VIII space-time. Mishra [14] has constructed the non- static plane symmetric Zeldovich fluid model in this theory with a time dependent gauge function. Recently, Mishra [15] has constructed static plane symmetric Zeldovich fluid model in scale invariant theory.

Rao et al. [16,17] have discussed cylindrically symmetric scalar meson fields and Brans-Dicke scalar fields. It is found from the literature that the scale invariant theory of gravitation has not been studied so far in Einstein-Rosen space-time. Hence, in this paper, we have taken an attempt to study the cylindrically symmetric space-time in the scale invariant theory of gravitation. A cosmological model has been presented.

\section{Field Equations}

Wesson [11,12] formulated a scale invariant theory of gravitation using a gauge function $\beta\left(x^{i}\right)$, where, $x^{i}, i=1,2,3,4$ are coordinates in the four-dimensional space-time and the tensor field is identified with the metric tensor $g_{i j}$. This theory is both coordinate and scale invariant in nature. The field equations formulated by Wesson $[11,12]$ for the combined scalar and tensor fields are:

$$
G_{i j}+2 \frac{\beta_{i j}}{\beta}-4 \frac{\beta_{, i} \beta_{, j}}{\beta^{2}}+\left(g^{a b} \frac{\beta_{, a} \beta_{, b}}{\beta^{2}}-2 g^{a b} \frac{\beta_{; a b}}{\beta}\right) g_{i j}=-\kappa T_{i j}
$$

with

$$
G_{i j} \equiv R_{i j}-\frac{1}{2} R g_{i j}
$$

Here, $G_{i j}$ is the conventional Einstein tensor involving 
$g_{i j}$. Semicolon and comma respectively denote covariant differentiation with respect to $g_{i j}$ and partial differentiation with respect to coordinates. The cosmological term $\Lambda g_{i j}$ of Einstein theory is transformed to $\Lambda_{0} \beta^{2} g_{i j}$ in scale invariant theory with a dimensionless constant $\Lambda_{0} . T_{i j}$ is the energy momentum tensor of the matter field and $\kappa=\frac{8 \pi G}{c^{4}}$.

The line element for Einstein- Rosen metric with a gauge function $\beta=\beta(c t)$ is.

with

$$
d s_{W}^{2}=\beta^{2} d s_{E}^{2}
$$

$$
d s_{E}^{2}=e^{2 A-2 B}\left(c^{2} d t^{2}-d r^{2}\right)-r^{2} e^{-2 B} d \varphi^{2}-e^{2 B} d z^{2}
$$

where $\mathrm{A}$ and $\mathrm{B}$ are functions of $\mathrm{t}$ only, and $\mathrm{c}$ is the velocity of light. Here we intend to build cosmological models in this space-time with a perfect fluid having the energy momentum tensor of the form

$$
T_{i j}^{m}=\left(p_{m}+\rho_{m} c^{2}\right) U_{i} U_{j}-p_{m} g_{i j}
$$

together with $g_{i j} U^{i} U^{j}=1$

where $U^{i}$ is the four-velocity vector of the fluid; $p_{m}$ and $p_{m}$ are the proper isotropic pressure and energy density of the matter respectively.

The non - vanishing components of conventional Einstein's tensor (2) for the metric (4) can be obtained as

$$
\begin{gathered}
G_{11}=\frac{1}{c^{2}}\left[B_{4}^{2}\right] \\
G_{14}=-\frac{1}{r}\left[A_{4}\right] \\
G_{22}=\frac{1}{c^{2}}\left[A_{44}+B_{4}^{2}\right] \\
G_{33}=\frac{1}{c^{2}}\left[A_{44}-2 B_{44}+B_{4}^{2}\right] \\
G_{44}=\left[B_{4}^{2}\right]
\end{gathered}
$$

Here afterwards the suffix 4 after a field variable denotes exact differentiation with respect to time $t$ only.

Using the comoving coordinate frame where $U^{i}=\delta_{4}^{i}$, the non-vanishing components of the field Equation (1) for the metric (3) can be written in the following explicit form:

$$
\begin{gathered}
G_{11}= \\
-\kappa p_{m} e^{2 A-2 B}-\frac{1}{c^{2}}\left[2 \frac{\beta_{44}}{\beta}-\frac{\beta_{4}^{2}}{\beta^{2}}+2 \frac{\beta_{4}}{\beta}\left(-A_{4}+B_{4}\right)-\Lambda_{0} \beta^{2} c^{2} e^{2 A-2 B}\right] \\
G_{14}=0
\end{gathered}
$$

i.e. $A=k_{1}$, where $k_{1}$ is an integrating constant.

$$
\begin{aligned}
& G_{22}= \\
& -\kappa p_{m} e^{2 A-2 B}-\frac{1}{c^{2}}\left[2 \frac{\beta_{44}}{\beta}-\frac{\beta_{4}^{2}}{\beta^{2}}+2 \frac{\beta_{4}}{\beta}\left(B_{4}\right)-\Lambda_{0} \beta^{2} c^{2} e^{2 A-2 B}\right]
\end{aligned}
$$

$G_{33}=$

$-\kappa p_{m} e^{2 A-2 B}-\frac{1}{c^{2}}\left[2 \frac{\beta_{44}}{\beta}-\frac{\beta_{4}^{2}}{\beta^{2}}-2 \frac{\beta_{4}}{\beta}\left(B_{4}\right)-\Lambda_{0} \beta^{2} c^{2} e^{2 A-2 B}\right]$

$G_{44}=$

$-\kappa \rho_{m} c^{4} e^{2 A-2 B}+\left[3 \frac{\beta_{4}^{2}}{\beta^{2}}-2 \frac{\beta_{4}}{\beta}\left(B_{4}\right)-\Lambda_{0} \beta^{2} c^{2} e^{2 A-2 B}\right]$

Equation (12) reduces the above set of Equations (11)-(15) as

$$
\begin{gathered}
G_{11}=G_{22}= \\
-\kappa p_{m} e^{2 k_{1}-2 B}-\frac{1}{c^{2}}\left[2 \frac{\beta_{44}}{\beta}-\frac{\beta_{4}^{2}}{\beta^{2}}+2 \frac{\beta_{4}}{\beta}\left(B_{4}\right)-\Lambda_{0} \beta^{2} c^{2} e^{2 k_{1}-2 B}\right] \\
G_{33}=-\kappa p_{m} e^{2 k_{1}-2 B}- \\
\frac{1}{c^{2}}\left[2 \frac{\beta_{44}}{\beta}-\frac{\beta_{4}^{2}}{\beta^{2}}-2 \frac{\beta_{4}}{\beta}\left(B_{4}\right)-\Lambda_{0} \beta^{2} c^{2} e^{2 k_{1}-2 B}\right] \\
G_{44}=-\kappa \rho_{m} c^{4} e^{2 k_{1}-2 B}+ \\
{\left[3 \frac{\beta_{4}^{2}}{\beta^{2}}-2 \frac{\beta_{4}}{\beta}\left(B_{4}\right)-\Lambda_{0} \beta^{2} c^{2} e^{2 k_{1}-2 B}\right]}
\end{gathered}
$$

Now, Equation (1) and Equations (16)-(18) (Wesson [12]) suggest the definitions of quantities $p_{v}$ (vacuum pressure) and $p_{v}$ (vacuum density) that involves neither the Einstein tensor of conventional theory nor the properties of conventional matter. These two quantities can be obtained as:

$$
\begin{gathered}
2 \frac{\beta_{44}}{\beta}-\frac{\beta_{4}^{2}}{\beta^{2}}+2 \frac{\beta_{4}}{\beta}\left(B_{4}\right)-\Lambda_{0} \beta^{2} c^{2} e^{2 k_{1}-2 B}=\kappa p_{v} c^{2} \\
2 \frac{\beta_{44}}{\beta}-\frac{\beta_{4}^{2}}{\beta^{2}}-2 \frac{\beta_{4}}{\beta}\left(B_{4}\right)-\Lambda_{0} \beta^{2} c^{2} e^{2 k_{1}-2 B}=\kappa p_{v} c^{2} \\
3 \frac{\beta_{4}^{2}}{\beta^{2}}-2 \frac{\beta_{4}}{\beta}\left(B_{4}\right)-\Lambda_{0} \beta^{2} c^{2} e^{2 k_{1}-2 B}=\kappa \rho_{v} c^{4}
\end{gathered}
$$

It is evident from Equations (19) and (20) that

$$
B_{4}=0 \Rightarrow B=k_{2} \text { since } \frac{\beta_{4}}{\beta} \neq 0
$$

where $k_{2}$ is an integrating constant. Using Equation (22) in Equations (19)-(21), the pressure and energy density for vacuum case can be obtained as 


$$
\begin{gathered}
p_{v}=\frac{1}{\kappa c^{2} e^{2 k_{1}-2 k_{2}}}\left[2 \frac{\beta_{44}}{\beta}-\frac{\beta_{4}^{2}}{\beta^{2}}+\Lambda_{0} \beta^{2} c^{2} e^{2 k_{1}-2 k_{2}}\right] \\
\rho_{v}=\frac{1}{\kappa c^{4} e^{2 k_{1}-2 k_{2}}}\left[3 \frac{\beta_{4}^{2}}{\beta^{2}}-\Lambda_{0} \beta^{2} c^{2} e^{2 k_{1}-2 k_{2}}\right]
\end{gathered}
$$

Here $p_{v}$ and $p_{v}$ relate to the properties of vacuum only in conventional physics. The definition of above quantities is natural as regards to the scale invariant properties of the vacuum. The total pressure and energy density can be defined as

$$
\begin{gathered}
p_{t} \equiv p_{m}+p_{v} \\
\rho_{t} \equiv \rho_{m}+\rho_{v}
\end{gathered}
$$

Using the aforesaid definitions of $p_{t}$ and $p_{t}$, the field equations in scale invariant theory i.e. (16)-(18) can now be written by using the components of Einstein tensor (6)-(10) and the results obtained in Equations (22)(24) as:

$$
\begin{gathered}
B_{4}^{2}=-\kappa p_{t} c^{2} e^{2 k_{1}-2 B} \\
-2 B_{44}+B_{4}^{2}=-\kappa p_{t} c^{2} e^{2 k_{1}-2 B} \\
B_{4}^{2}=-\kappa \rho_{t} c^{4} e^{2 k_{1}-2 B}
\end{gathered}
$$

\section{Solution}

From Equations (27) and (29), we obtained the equation of state

$$
p_{t}=\rho_{t} c^{2}
$$

Using Equation (27) in Equation (28), we obtained

$$
B=d_{1} t+d_{2}
$$

where $d_{1}$ and $d_{2}$ are integrating constants.

Substituting Equation (31) in Equation (27) and Equation (29) respectively, the total pressure ' $p_{t}$ ' and energy density ' $\rho_{t}$ ' can be obtained as:

$$
p_{t}=\rho_{t} c^{2}=\frac{1}{\kappa c^{2}}\left[-\frac{d_{1}^{2}}{q e^{-2 d_{1} t}}\right]
$$

where $q=e^{2 k_{1}-2 k_{2}}$ is a constant. The reality condition demands that $d_{1}^{2}<0$.

Using Equation (31) in Equations (23) and (24) respectively and taking $\beta=\frac{1}{c t}$, the pressure and energy density corresponding to vacuum case can be calculated as:

$$
\begin{aligned}
& p_{v}=-\frac{1}{\kappa c^{2} q}\left[\frac{\Lambda_{0} q-1}{t^{2}}\right] \\
& \rho_{v}=\frac{1}{\kappa c^{4} q}\left[\frac{\Lambda_{0} q-3}{t^{2}}\right]
\end{aligned}
$$

In this case, when there is no matter and the gauge function $\beta$ is a constant, one recovers the relation $c^{2} \rho_{v}=c^{4} \frac{\Lambda_{G R}}{8 \pi G}=-p_{v}$ i.e. $c^{2} \rho_{v}+p_{v}=0$, which is the equation of state for vacuum. Here $\Lambda_{G R}=\Lambda_{0} \beta^{2}=$ constant, is the cosmological constant in general relativity. Also $p_{v}$ being dependent on the constants $\Lambda_{G R}, c$ and $G$, is uniform in all directions and hence isotropic in nature. The cosmological model with this equation of state is rare in literature and is known as $\rho$ - vacuum or false vacuum or degenerate vacuum model [18-21], the corresponding model in the static case is a well known de-Sitter model.

Now the matter pressure and density can be obtained as:

$$
\begin{aligned}
& p_{m}=p_{t}-p_{v}=\frac{1}{\kappa c^{2}}\left[\frac{\Lambda_{0} q-1}{q t^{2}}-\frac{d_{1}^{2}}{e^{2 k_{1}-2 d_{1} t-d_{2}}}\right] \\
& \rho_{m}=\rho_{t}-\rho_{v}=\frac{1}{\kappa c^{4}}\left[\frac{3-\Lambda_{0} q}{q t^{2}}-\frac{d_{1}^{2}}{e^{2 k_{1}-2 d_{1} t-d_{2}}}\right]
\end{aligned}
$$

Now, we have $\rho_{m} \rightarrow \infty$ as $t \rightarrow 0$ and $\rho_{m} \rightarrow \infty$ as $t \rightarrow \infty$. Also when $t<0, \rho_{m} \rightarrow$ constant. It is interesting to note that the model free from singularity.

So, the Einstein-Rosen cylindrically symmetric model in scale invariant theory of gravitation is given by the Equations (12), (31) and (32) and the metric in this case is

$d S_{W}^{2}=$

$\frac{1}{(c t)^{2}}\left[e^{2 k_{1}-2 d_{1} t-2 d_{2}}\left(c^{2} d t^{2}-d r^{2}\right)-r^{2} e^{-2 d_{1} t-2 d_{2}} d \varphi^{2}-e^{2 d_{1} t+2 d_{2}} d z^{2}\right]$

\section{Some Physical Properties of the Model}

The scalar expansion, $\theta=U_{; i}^{i}=3 \frac{Q_{T}}{Q}$ for the model given by Equation (37) takes the form

$$
\theta=-\frac{1}{C}\left(d_{1} e^{d_{1} t+d_{2}-k_{1}}\right)
$$

Thus, we find $\theta \rightarrow-\frac{1}{c}\left(d_{1} e^{d_{2}-k_{1}}\right)$ as $t \rightarrow 0$ and $\theta \rightarrow 0$ as $t \rightarrow \infty$.

If $c>0, d_{1}<0$ and $d_{2}>k_{1}$ the model represents expanding one for $t<t_{1}\left(=\frac{k_{1}-d_{2}}{d_{1}}\right)$.

It is also observed that as $\frac{\rho_{m}}{\theta^{2}} \rightarrow$ constant as $t \rightarrow \infty$ and $\frac{\rho_{m}}{\theta^{2}} \rightarrow \theta$ as $t \rightarrow 0$. Thus the universe confirms the homogeneity nature of the space-time. 
Following Raychaudhuri [22], the anisotropy $|\sigma|$ can be defined as

$$
\begin{aligned}
& \sigma^{2}= \\
& {\left[\left(\frac{g_{11,4}}{g_{11}}-\frac{g_{22,4}}{g_{22}}\right)^{2}+\left(\frac{g_{22,4}}{g_{22}}-\frac{g_{33,4}}{g_{33}}\right)^{2}+\left(\frac{g_{33,4}}{g_{33}}-\frac{g_{11,4}}{g_{11}}\right)^{2}\right]}
\end{aligned}
$$

Consequently for the model $(37), \sigma^{2}=\frac{8}{3}\left(d_{1} t+d_{2}\right) \neq 0$. So the shear scalar remains constant for $t \rightarrow 0$ and becomes indefinitely large for $t \rightarrow \infty$.

The ratio of anisotropy to expansion $\frac{\sigma^{2}}{\theta^{2}}=$ $\frac{8 c^{2}}{3} e^{2 k_{1}-2 d_{2}} \neq 0$ for $t=0$. Thus there is a singularity of $t=0$ for $2 k_{1}-2 d_{2}$ is not very large. Moreover, the model is isotropy for finite $t$ and does not approach isotropy for large value of $t$.

It is observed that the vorticity ' $w$ ' vanishes which indicates that $u^{i}$ is hypersurface orthogonal. As the acceleration $\dot{u}_{i}$ found to be zero, the matter particle follows geodesic path in this theory.

\section{Conclusions}

Every physical theory carries its own mathematical structure and the validity of the theory is usually studied through the exact solution of the mathematical structure. In this theory black holes do not appear to exist. If the existence of black holes in nature is confirmed, it will represent a great success of general theory of relativity. Since there is no concrete evidence at present for the existence of black holes, one can take a stand point that black holes represents a familiar concept of space time. Therefore the scale invariant theory involves gauge theories as it relates to gravitational theories with an added scalar field.

The significance of the present work deals with the modification of gravitational and geometrical aspects of Einstein's equations. These are 1) scale invariant theory of gravitation which describes the interaction between matter and gravitation in scale free manner; and 2) the gauge transformation, which represents a change of units of measurements and hence gives a general scaling of physical system. The nature of the cosmological model with modified gravity that would reproduce the kinematical history and evolution of perturbation of the universe is investigated.

Here, cylindrically symmetric static zeldovich fluid model is obtained in the presence of perfect fluid distribution in scale invariant theory of gravitation. As far as matter is concerned the model does not admit either big bang or big crunch during evolution till infinite future. The model appears to be a steady state.

\section{Acknowledgements}

The authors are very much grateful to the referee for his valuable suggestions for the improvement of the paper.

\section{References}

[1] C. H. Brans and R. H. Dicke, "Mach's Principle and a Relativistic Theory of Gravitation," Physical Review A, Vol. 124, No. 3, 1961, pp. 925-935.

[2] K. Nordverdt, Jr., "Post Newtonian Metric for a General Class of Scalar-Tensor Gravitational Theories and Observational Consequences," The Astrophysical Journal, Vol. 161, 1970, pp. 1059-1067.

[3] R. V. Wagoner, "Scalar-Tensor Theory and Gravitational Waves", Physical Review D, Vol. 1, No. 2, 1970, pp. 3209-3216.

[4] D. K. Ross, "Scalar-Tensor Theory of Gravitation," Physical Review D, Vol. 5, No. 2, 1972, pp. 284-192.

[5] K. A. Dunn, "A Scalar-Tensor Theory of Gravitation," Journal of Mathematical Physics, Vol. 15, No. 12, 1974, pp. 2229-2231.

[6] D. Saez and V. J. Ballester, "A Simple Coupling with Cosmological Implications," Physics Letters A, Vol. A113, No. 9, 1985, pp. 467-470.

[7] V. Canuto, S. H. Hseih and P. J. Adams, "Scale -Covariant Theory of Gravitation and Astrophysical Applications," Physics Review Letters, Vol. 39, No. 88, 1977, pp. 429- 432

[8] P. A. M. Dirac, "Long Range Forces and Broken Symmetries," Proceedings of the Royal Society of London, Vol. A333, 1973, pp. 403-418.

[9] P. A. M. Dirac, "Cosmological Models and the Large Number Hypothesis," Proceedings of the Royal Society of London, Vol. A338, No. 1615, 1974, pp. 439-446.

[10] F. Hoyle and J. V. Narlikar, "Action at a Distance in Physics and Cosmology," W. H. Freeman, San Francisco, 1974.

[11] P. S. Wesson, "Gravity, Particle and Astrophysics," D. Reidel, Dordrecht, 1980.

[12] P. S. Wesson, "Scale-Invariant Gravity-A Reformulation and an Astrophysical Test," Monthly Notices of the Royal Astronomical Society, Vol. 197, 1981, pp. 157-165.

[13] G. Mohanty and B. Mishra, "Scale Invariant Theory for Bianchi Type VIII and IX Space-times with Perfect Fluid," Astrophysics and Space Science, Vol. 283, No. 1, 2003, pp. 67-74.

[14] B. Mishra, "Non-Static Plane Symmetric Zeldovich Fluid Model in Scale Invariant Theory," Chinese Physics Letters, Vol. 21, No. 12, 2004, pp. 2359-2361.

[15] B. Mishra, "Static Plane Symmetric Zeldovich Fluid Model in Scale Invariant Theory," Turkish Journal of Physics, Vol. 32, No. 6, 2008, pp. 357-361. 
[16] J. R. Rao, A. R. Roy and R. N. Tiwari, "A Class of Exact Solutions for Coupled Electromagnetic and Scalar Fields for Einstein Rosen Metric I," Annals of Physics, Vol. 69, No. 2, 1972, pp. 473-486.

[17] J. R. Rao, R. N. Tiwari and K. S. Bhamra, "Cylindrical Symmetric Brans-Dicke Fields II", Annals of Physics, Vol. 87, 1974, pp. 480-497.

[18] J. J. Bloome and W. Priester, "Urknall und Evolution Des. Kosmos II," Naturewissenshaften, Vol. 2, 1984, pp. 528531.
[19] P. C. W. Davies, "Mining the Universe," Physical Review D, Vol. 30, No. 4, 1984, pp. 737-742.

[20] C. J. Hogan, "Microwave Background Anisotropy and Hydrodynamic Formation of Large-Scale Structure," Astrophysical Journal, Vol. 310, 1984, p. 365.

[21] N. Kaiser and A. Stebbins, "Microwave Anisotropy Due to Cosmic Strings," Nature, Vol. 310, No. 5976, 1984, pp. 391- 393.

[22] A. Raychaudhuri, Theoretical Cosmology, Oxford University Press, 1979. 